EGU2020-10874

https://doi.org/10.5194/egusphere-egu2020-10874

EGU General Assembly 2020

(c) Author(s) 2020. This work is distributed under

the Creative Commons Attribution 4.0 License.

\title{
A posteriori verification methodology for astrochronology: a step further to improve the falsifiability of cyclostratigraphy
}

\author{
Sébastien Wouters ${ }^{1,2}$, Michel Crucifix ${ }^{3}$, Matthias Sinnesael ${ }^{4}$, Anne-Christine Da Silva ${ }^{1}$, Christian \\ Zeeden $^{5}$, Miroslav Zivanovic ${ }^{6}$, Frédéric Boulvain ${ }^{1}$, and Xavier Devleeschouwer ${ }^{2}$ \\ ${ }^{1}$ Petrosed, ULiege, Liege, Belgium (sebastien.wouters@doct.uliege.be) \\ ${ }^{2}$ O.D. Earth and History of Life, Royal Belgian Institute of Natural Sciences, Brussels, Belgium \\ ${ }^{3}$ Earth \& Climate, Université Catholique de Louvain, Louvain-La-Neuve, Belgium \\ ${ }^{4}$ Analytical, Environmental and Geo-Chemistry, Vrije Universiteit Brussel, Brussels, Belgium \\ ${ }^{5}$ Leibniz Institute for Applied Geophysics, Geozentrum Hannover, Hannover, Germany \\ ${ }^{6}$ Department of Electrical and Electronic Engineering, Universidad Pública de Navarra, Pamplona, Spain
}

Cyclostratigraphy is increasingly used to improve the Geologic Time Scale and our understanding of past climatic systems. However, except in a few existing methodologies, the quality of the results is often not evaluated.

We propose a new methodology to document this quality, through a decomposition of a signal into a set of narrow band components from which instantaneous frequency and amplitude can be computed, using the Hilbert transform. The components can be obtained by Empirical Mode Decomposition (EMD), but also by filtering a signal (be it tuned or not) in any relevant way, and by subsequently performing EMD on the signal minus its filtered parts.

From that decomposition, verification is performed to estimate the pertinence of the results, based on different concepts that we introduce:

- Integrity quantifies to what extent the sum of the components is equal to the signal. It is defined as the cumulated difference between (1) the signal, and (2) the summed components of the decomposition. EMD fulfils integrity by design, except for errors caused by floatingdecimal arithmetic. Ensemble Empirical Mode Decomposition (EEMD) may fail to satisfy integrity unless noisy realisations are carefully chosen in the algorithm to cancel each other when averaging the realisations. We present such an algorithm implemented in R: "extricate", which performs EEMD in a few seconds.

- Parsimony checks that the decomposition does not generate components that heavily cancel out. We propose to quantify it as the ratio between (1) the cumulated absolute values of each component (except the trend), and (2) the cumulated absolute values of the signal (minus the trend). The trend should be ignored in the calculation, because an added trend decreases the parsimony estimation of a similar decomposition.

- IMF departure (IMFD) quantifies the departure of each component to the definition of intrinsic 
mode functions (IMF), from which instantaneous frequency can reliably be computed. We define it as the mean of the absolute differences of the base 2 logarithms of frequencies obtained using (1) a robust generalized zero-crossing method (GZC, which simplifies the components into extrema separated by zero-crossings) and (2) a more local method such as the Hilbert Transform.

- Reversibility is the concept that all initial data points are preserved, even after linear interpolation and tuning. This allows to revert back to the original signal and discuss the significance of each data point. To facilitate reversibility we introduce the concept of quanta (smallest depth or time interval having significance for a given sampling) and an algorithm computing the highest rational common divisor of given values in R: "divisor".

This new methodology allows to check the final result of cyclostratigraphic analysis independently of how it was performed (i.e. a posteriori). Once the above-mentioned concepts are taken into account, the instantaneous frequencies, ratios of frequencies and amplitudes of the components can be computed and used to interpret the pertinence of the analysis in a geologically meaningful way. The instantaneity and independence of frequency and amplitude so obtained open a new way of performing time-series analysis. 